\title{
Surgeon's protection during ophthalmic surgery in the Covid-19 era: a novel fitted drape for ophthalmic operating microscopes
}

\author{
Rodrigo Anguita ${ }^{1} \cdot$ Harry Tossounis ${ }^{1} \cdot$ Manjit Mehat $^{1} \cdot \operatorname{lan}$ Eames $^{2} \cdot$ Louisa Wickham $^{1}$
}

Received: 14 April 2020 / Revised: 22 April 2020 / Accepted: 23 April 2020 / Published online: 7 May 2020

(c) The Royal College of Ophthalmologists 2020

In the current period of COVID-19 pandemic, healthcare professionals are faced with the challenge of minimising the risk of viral transmission. Ophthalmologists rely on close patient contact to examine and treat ocular disease, resulting in the exposure of naso-pharyngeal flow and dispersion of viral droplets. This is of particular concern when patient contact time is prolonged, such as when performing urgent or emergency surgery. Moreover, surgical procedures, including pars plana vitrectomy, that involve high-speed devices are considered to be aerosol generating procedures (https://www.rcophth.ac.uk/w p-content/uploads/2020/04/Vitreoretinal-surgery-managementguidance-070420.pdf). Although the viral load in conjunctiva and tears is thought to be low [1], viral load in the vitreous and the potential of viral transmission remains unknown. Literature on the risks of aerosol transmission of infection in hospital operating theatres is well described [2]. Exhaled air plumes from a patient lying on a bed can be spread over long distances, assisted by differences in air temperature and density [3].

\section{Materials}

- Clear monitor cover found at ALCON vitrectomy prepack $\left(3 \mathrm{M}^{\oplus}\right)$.

- Semi-transparent ophthalmic drape.

- Sterile steri-drape $1040(35 \times 35 \mathrm{~cm})\left(3 \mathrm{M}^{\circledast}\right)$.

- Sterile scissors.

The novel fitted ophthalmic microscope drape is produced under sterile conditions within the operating theatre. A sterile cover is laid on top of a surgical trolley. The ALCON vitrectomy prepack is opened and the clear plastic cover for

Rodrigo Anguita

rodrigoanguita@gmail.com

1 Vitreoretinal department, Moorfields Eye Hospital NHS Foundation Trust, London, UK

2 Department of Mechanical Engineering, UCL, London, UK
ALCON Constellation machine monitor is removed and unfolded on the sterile trolley. A rectangle shape measuring $\sim 40 \times 40 \mathrm{~cm}$ is cut out using scissors and put to one side (Fig. 1a). The next step is the preparation of the microscope drape. For this, we use a commercially available semitransparent ophthalmic surgery drape. The drape is unfolded on the trolley and the fluid collection bag is cut away (Fig. 1b). The drape is then positioned with the adhesive square section (the part that ordinarily adheres to the patients' eye) facing up. It is this adhesive square that will be attached to the operating microscope. The previously prepared rectangle clear monitor cover cut out is positioned on top of the unfolded semi-transparent ophthalmic drape, about $10 \mathrm{~cm}$ below the non-stick paper edge, and is attached to it with surgical tape at each of the four sides (Fig. 1c). This section of the ophthalmic drape is then folded over and the semi-opaque drape overlying the transparent rectangle cut away with scissors. This results in a semi-transparent ophthalmic drape with a transparent window made by the monitor cover cut out, to allow direct visualisation of the surgical field. (Fig. 1d final product). The final step is to cut a cross in the adhesive square of the drape in order to adhere the drape to the microscope. The drape is then ready to fix into position once the BIOM plate has been screwed into place (Fig. 1e).

The patient is positioned on the Stryker ${ }^{\circledast}$ bed and is draped as normal. A Stryker ${ }^{\circledR}$ ophthalmic wrist rest is adjusted on the bed. The standard drape is then pushed through the gap between the head rest and the wrist rest to create a sterile drape pocket for placement of surgical instruments. This is an important step which creates a sterile pocket to house and safeguard instruments under the microscope fitted drape during the surgery (Fig. 1f).

The Biom ${ }^{\circledast}$ plate is secured on the ophthalmic microscope and with the aid of the scrub nurse the surgeon fits the prepared ophthalmic microscope drape around the rim of the microscope lens, being mindful to position the transparent window in the direction of the operating surgeon. The rest of the drape then hangs over the surgical field in a 

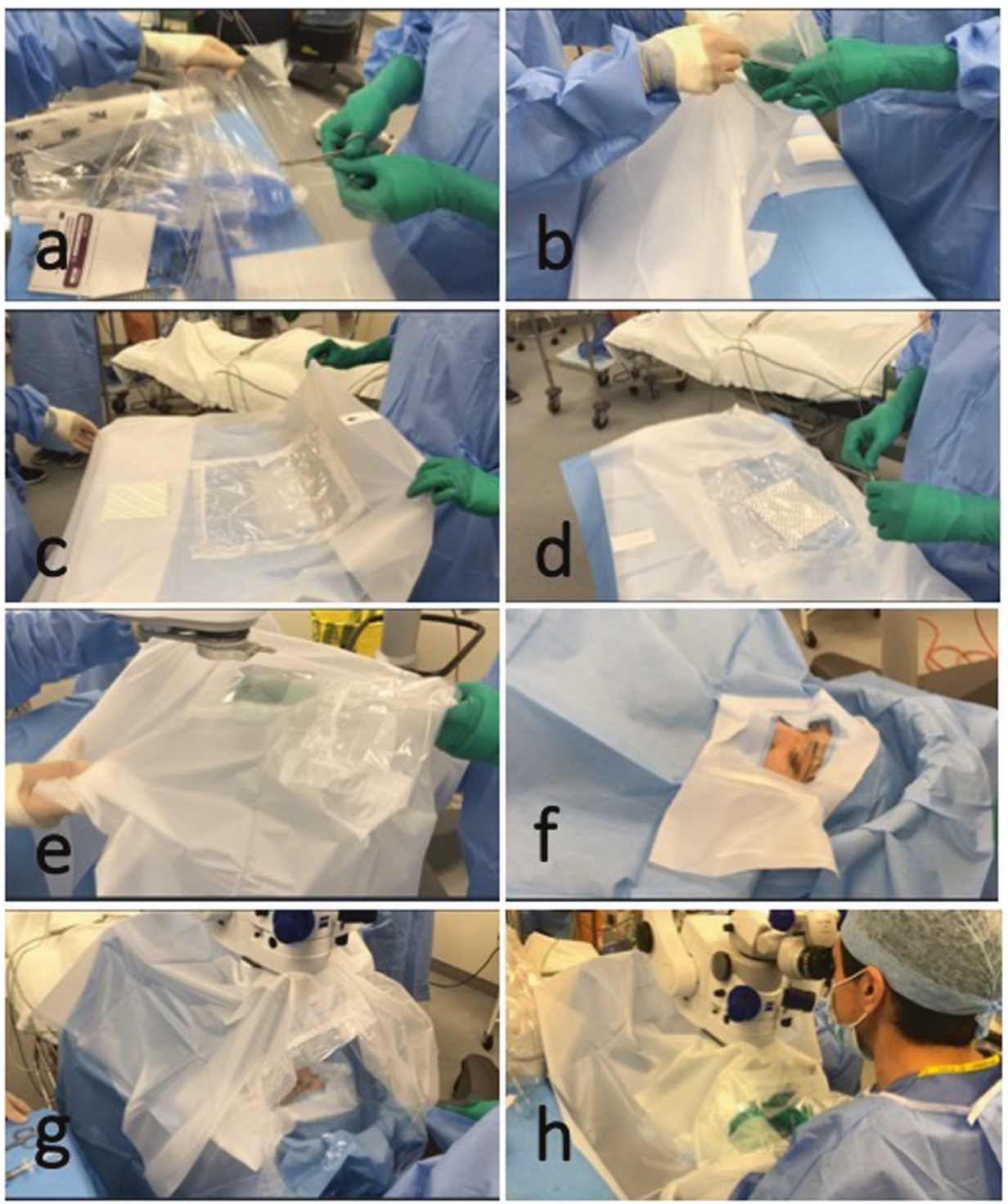

Fig. 1 Serial colour photographs illustration construction of the novel fitted drape for ophthalmic microscopes. a A rectangle shape measuring $\sim 40 \times 40 \mathrm{~cm}$ is cut out from a sterile transparent monitor cover for the ALCON Constellation machine, using scissors. b Commercially available semi-transparent ophthalmic surgery drape is unfolded on a trolley and the fluid collection bag is cut away. c The drape is positioned with the adhesive side of the square facing upwards. The previously prepared clear monitor cover cut out (a) is positioned on top of the unfolded ophthalmic drape, about $10 \mathrm{~cm}$ below the edge of the adhesive square, and is attached to it with surgical tape at each of the four sides. d The ophthalmic drape is then turned over and the ophthalmic surgery drape, overlying the transparent rectangle is cut

pyramid shape fashion protecting the surgeon from aerosol products generated during the procedure whilst ensuring that there is good visualisation and access to the surgical instruments and operating field. (Fig. 1g, h).

When removing the microscope drape, the drape should be first detached from the microscope rim and gently lowered on to the patient surgical drape below. The microscope drape

away with scissors. e A cross is cut into the adhesive square of the drape in order to adhere the drape to the microscope. The Biom ${ }^{\circledast}$ plate is secured on the ophthalmic microscope and with the aid of the scrub nurse the surgeon fits the prepared ophthalmic microscope drape around the rim of the microscope lens, with the transparent window facing the direction of the operating surgeon. $f$ The patient is positioned on the Stryker bed and is draped as normal. A Stryker ophthalmic wrist rest is adjusted on the bed. The standard ophthalmic drape is then pushed through the gap between the head rest and the wrist rest to create a sterile drape pocket for placement of surgical instruments. $\mathbf{g}, \mathbf{h}$ The microscope with the attached fitted drape is positioned over the surgical fields to create a pyramid shape.

should then be removed together with the patient drape to reduce the risk of dispersing particles and the residue of dried droplets on the surface of the patient drape.

This is a simple and efficient method to reduce the risk of aerosol spread within theatres. It is important to highlight that this drape should not replace the use of adequate PPE (respirator masks FFP3 and eye protection), however the 
use of additional drape will allow us to mitigate against risk of intraoperative spread of aerosol particles across the theatre where they may deposit themselves onto theatre staff and surfaces.

\section{Compliance with ethical standards}

Conflict of interest The authors declare that they have no conflict of interest.

Publisher's note Springer Nature remains neutral with regard to jurisdictional claims in published maps and institutional affiliations.

\section{References}

1. Xia J, Tong J, Liu M, Shen Y, Guo D 2020. Evaluation of coronavirus in tears and conjunc-tival secretions of patients with SARS-CoV-2 infection. J Med Virol. https://doi.org/10.1002/jmv. 25725.

2. Tang JW, Li Y, Eames I, Chan PKS, Ridgway GL. Factors involved in the aerosol transmission of infection and control of ventilation in healthcare premises. $J$ Hospital Infect. 2006;64:100-14.

3. Qian H, Li Y, Nielsen PV, Hyldgaard CE, Wong TW, Chwang ATY. Dispersion of exhaled droplet nuclei in a two-bed hospital ward with three different ventilation systems. Indoor Air. 2006;16:111-28. 